\title{
Preparation of $\mathrm{NiS} / \mathrm{ZnIn}_{2} \mathrm{~S}_{4}$ as a superior photocatalyst for hydrogen evolution under visible light irradiation
}

\author{
Liang Wei, Yongjuan Chen, Jialin Zhao and Zhaohui Li ${ }^{*}$
}

\section{Full Research Paper}

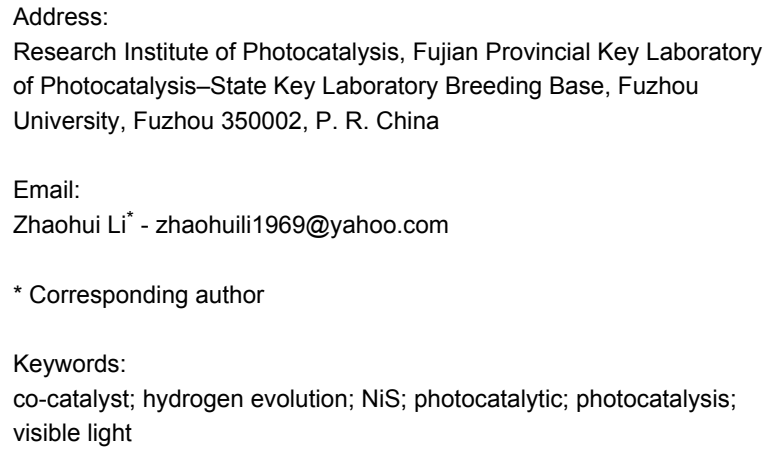

Research Institute of Photocatalysis, Fujian Provincial Key Laboratory of Photocatalysis-State Key Laboratory Breeding Base, Fuzhou University, Fuzhou 350002, P. R. China

Email:

Zhaohui Li* - zhaohuili1969@yahoo.com

* Corresponding author

Keywords:

co-catalyst; hydrogen evolution; NiS; photocatalytic; photocatalysis; visible light

\author{
Beilstein J. Nanotechnol. 2013, 4, 949-955. \\ doi:10.3762/bjnano.4.107 \\ Received: 14 October 2013 \\ Accepted: 16 December 2013 \\ Published: 23 December 2013 \\ This article is part of the Thematic Series "Photocatalysis". \\ Guest Editor: R. Xu \\ (C) 2013 Wei et al; licensee Beilstein-Institut. \\ License and terms: see end of document.
}

\begin{abstract}
In this study, $\mathrm{NiS} / \mathrm{ZnIn}_{2} \mathrm{~S}_{4}$ nanocomposites were successfully prepared via a facile two-step hydrothermal process. The as-prepared samples were characterized by X-ray diffraction (XRD), X-ray photoelectron spectroscopy (XPS), transmission electron microscopy (TEM) and high-resolution transmission electron microscopy (HRTEM). Their photocatalytic performance for hydrogen evolution under visible light irradiation was also investigated. It was found that the photocatalytic hydrogen evolution activity over hexagonal $\mathrm{ZnIn}_{2} \mathrm{~S}_{4}$ can be significantly increased by loading NiS as a co-catalyst. The formation of a good junction between $\mathrm{ZnIn}_{2} \mathrm{~S}_{4}$ and NiS via the two step hydrothermal processes is beneficial for the directional migration of the photo-excited electrons from $\mathrm{ZnIn}_{2} \mathrm{~S}_{4}$ to NiS. The highest photocatalytic hydrogen evolution rate $(104.7 \mu \mathrm{mol} / \mathrm{h})$, which is even higher than that over Pt/ZnIn ${ }_{2} \mathrm{~S}_{4}$ nanocomposite $(77.8 \mu \mathrm{mol} / \mathrm{h})$, was observed over an optimum NiS loading amount of $0.5 \mathrm{wt} \%$. This work demonstrates a high potential of the developing of environmental friendly, cheap noble-metal-free co-catalyst for semiconductor-based photocatalytic hydrogen evolution.
\end{abstract}

\section{Introduction}

Hydrogen is a clean and green fuel. The conversion and store of solar energy in the form of hydrogen by photocatalytic water splitting holds great promise to meet the future energy and environment requirements [1-3]. Ever since the pioneering work of a photo-electrochemical cell using $\mathrm{Pt}_{-} \mathrm{TiO}_{2}$ electrodes for water splitting by Fujishima and Honda in 1972, great efforts have been devoted to the development of highly efficient semiconductor photocatalysts for hydrogen production [4]. So far, a variety of active photocatalysts for hydrogen production, including metal oxides [5-8], sulfides [9-11], oxynitrides [1214], as well as the metal-free semiconductors [15] have already been developed. 
Among the numerous types of semiconductor systems studied, metal sulfides have demonstrated promising activities towards hydrogen evolution from water containing sacrificial reagents under visible light. $\mathrm{ZnIn}_{2} \mathrm{~S}_{4}$ is a ternary chalcogenide with a suitable band gap (2.34-2.48 eV) well corresponding to the visible light absorption. $\mathrm{ZnIn}_{2} \mathrm{~S}_{4}$ exhibits two distinct polymorphs based on cubic and hexagonal lattices [16], which can be controlled synthesized via a facile hydrothermal method using different precursors. Previous studies revealed that both polymorphs of $\mathrm{ZnIn}_{2} \mathrm{~S}_{4}$ are active for photocatalytic hydrogen generation under visible light irradiations and show considerable chemical stability [17-19]. However, the photocatalytic hydrogen evolution activity over pure $\mathrm{ZnIn}_{2} \mathrm{~S}_{4}$ is low, due to the poor separation efficiency and migration ability of the photoexcited charge carriers. A variety of effects have been made to enhance the photocatalytic performance of $\mathrm{ZnIn}_{2} \mathrm{~S}_{4}$. For example, by size control on $\mathrm{ZnIn}_{2} \mathrm{~S}_{4}$ [20], doping with transition metals [21] and incorporation of metal sulfides [22] or RGO [23] into $\mathrm{ZnIn}_{2} \mathrm{~S}_{4}$ nanostructures, the photocatalytic performance for hydrogen evolution over $\mathrm{ZnIn}_{2} \mathrm{~S}_{4}$ have been enhanced to a certain degree.

Studies on semiconductor-based photocatalysts revealed that the deposition of a suitable co-catalyst on the semiconductor photocatalysts can play important roles in promoting their photocatalytic performance for hydrogen evolution. An appropriate co-catalyst can suppress the recombination of the photo-generated charge carriers, lower the overpotential for hydrogen evolution and also provide redox reaction sites for hydrogen evolution to avoid back reactions. Due to their negligible overpotential for hydrogen evolution and excellent kenetics for driving the hydrogen evolution reaction, noble metals like $\mathrm{Pt}$ [24-26], $\mathrm{Rh}$ [27], $\mathrm{Au}$ [28,29] and their oxides like $\mathrm{RuO}_{2}$ [30], $\mathrm{Rh}_{\mathrm{x}} \mathrm{Cr}_{2-\mathrm{x}} \mathrm{O}_{3}$ [31-33] are generally used as the co-catalysts for photocatalytic hydrogen evolution. However, the precious metals are expensive and to reduce the cost of renewable hydrogen evolution, it is necessary to explore alternative co-catalysts based on inexpensive transition metals.

Our recent studies revealed that $\mathrm{MoS}_{2}$, a good electrocatalyst for hydrogen evolution [34], can be an effective co-catalyst in promoting photocatalytic hydrogen evolution over $\mathrm{ZnIn}_{2} \mathrm{~S}_{4}$ and $\mathrm{MoS}_{2} / \mathrm{ZnIn}_{2} \mathrm{~S}_{4}$ show even superior performance for hydrogen evolution than $\mathrm{Pt} / \mathrm{ZnIn}_{2} \mathrm{~S}_{4}$ [35]. NiS, a p-type semiconductor, is also reported to be a good electrocatalyst for cathodic hydrogen evolution in water electrolysis [36]. Although $\mathrm{Ni}$ and $\mathrm{NiO}$ have already been used as co-catalysts for hydrogen evolution over oxide semiconductor photocatalysts, the application of $\mathrm{NiS}$ as co-catalyst for photocatalytic hydrogen evolution is less studied $[37,38]$. Only until recently, Xu et al. reported that NiS can be used as co-catalyst to enhance the photocatalytic hydrogen evolution over CdS [39]. It was found that NiS/CdS photocatalysts prepared via a simple hydrothermal loading method showed high photocatalytic activity for hydrogen evolution in the presence of lactic acid as sacrificial agent and a high quantum efficiency of $51.3 \%$ at $420 \mathrm{~nm}$ was obtained in this system. Later on, several other reports have also reported the using of NiS as a co-catalyst for photocatalytic hydrogen evolution [40-42].

Herein, we reported the preparation of $\mathrm{NiS} / \mathrm{ZnIn}_{2} \mathrm{~S}_{4}$ nanocomposites via a two-step hydrothermal method and its application for photocatalytic hydrogen evolution under visible light irradiation. It was found that $\mathrm{NiS}$ can be an effective co-catalyst for photocatalytic hydrogen evolution over $\mathrm{ZnIn}_{2} \mathrm{~S}_{4}$. The as-prepared $\mathrm{NiS} / \mathrm{ZnIn}_{2} \mathrm{~S}_{4}$ nanocomposites showed superior photocatalytic performance for hydrogen evolution under visible light irradiation and the activity of $\mathrm{NiS} / \mathrm{ZnIn}_{2} \mathrm{~S}_{4}$ with optimized amount of NiS is even higher than that of $\mathrm{Pt} / \mathrm{ZnIn}_{2} \mathrm{~S}_{4}$. A possible enhancement mechanism based on the co-catalyst and the formed junction for the improved photocatalytic activity in the $\mathrm{NiS} / \mathrm{ZnIn}_{2} \mathrm{~S}_{4}$ system was also proposed.

\section{Results and Discussion}

Figure 1 shows the XRD patterns of the as-prepared pure $\mathrm{ZnIn}_{2} \mathrm{~S}_{4}$ and sample with loading amount of $0.5 \mathrm{wt} \% \mathrm{NiS}$. As shown in Figure 1, all the samples showed diffraction peaks of 2 Theta values at $21.5^{\circ}, 27.6^{\circ}, 30.4^{\circ}, 39.8^{\circ}, 47.2^{\circ}, 52.4^{\circ}$ and $55.6^{\circ}$, which are corresponding to the (006), (102), (104), (108), (110), (116) and (022) crystallographic planes of hexagonal $\mathrm{ZnIn}_{2} \mathrm{~S}_{4}$ (JCPDS, No. 03-065-2023). This suggests that there is no obvious phase change in $\mathrm{ZnIn}_{2} \mathrm{~S}_{4}$ during the hydrothermal process in the preparation of the $\mathrm{NiS} / \mathrm{ZnIn}_{2} \mathrm{~S}_{4}$ nanocomposites. No characteristic diffraction peaks associated with NiS are

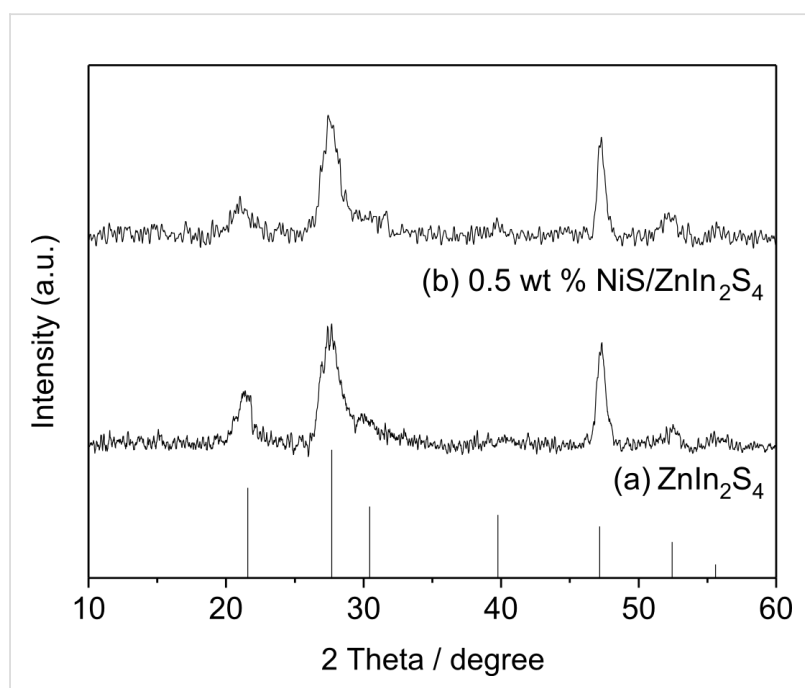

Figure 1: XRD patterns of (a) pure $Z n \ln _{2} \mathrm{~S}_{4}$; (b) $0.5 \mathrm{wt} \% \mathrm{NiS} / \mathrm{Zn} \mathrm{nn}_{2} \mathrm{~S}_{4}$. 
observed in these samples, probably due to the low amount of NiS loaded and its high dispersion on $\mathrm{ZnIn}_{2} \mathrm{~S}_{4}$.

Figure 2a shows the TEM image of our previous studies that the hydrothermally prepared $\mathrm{ZnIn}_{2} \mathrm{~S}_{4}$ sample was composed of microspheres with dimension in the range of 2-6 $\mu \mathrm{m}$ assembled by densely packed petals [16]. However, the TEM image of the current $\mathrm{NiS} / \mathrm{ZnIn}_{2} \mathrm{~S}_{4}$ sample shows that the $\mathrm{ZnIn}_{2} \mathrm{~S}_{4}$ microspheres were partially decomposed after the second hydrothermal process (Figure 2b). Although no characteristic diffraction peaks corresponding to NiS nanoparticles were observed in the XRD patterns, the existence of NiS in the nanocomposite is confirmed by the HRTEM image (Figure 2c). Clear lattice fringes of $0.32 \mathrm{~nm}$ and $0.29 \mathrm{~nm}$, which can be ascribed to the (102) plane of hexagonal $\mathrm{ZnIn}_{2} \mathrm{~S}_{4}$ and the (300) plane of rhombohedral NiS respectively, can be observed. As shown in Figure 2b, the NiS nanoparticle has a diameter of about $5 \mathrm{~nm}$ and directly contact with $\mathrm{ZnIn}_{2} \mathrm{~S}_{4}$. It is believed that the second hydrothermal treatment during the deposition of NiS on $\mathrm{ZnIn}_{2} \mathrm{~S}_{4}$ is important for the formation of a junction between these two components. The energy-dispersive X-ray spectrometry (EDS) analysis as shown in the inset of Figure 2c also confirms the existence of $\mathrm{Ni}$.

XPS analyses were carried out to evaluate the surface chemical composition and electronic state on $0.5 \mathrm{wt} \% \mathrm{NiS} / \mathrm{ZnIn}_{2} \mathrm{~S}_{4}$ sample, and the results are shown in Figure 3. The survey XPS spectrum, as shown in Figure 3a, confirms the existence of Zn, In, and $\mathrm{S}$. The high resolution XPS spectra of $\mathrm{S} 2 \mathrm{p}$ region can be deconvoluted into two peaks at around 161.7 and $162.8 \mathrm{eV}$, which can be assigned to $S 2 p_{3 / 2}$ and $S 2 p_{1 / 2}$ respectively in $S^{2-}$ (Figure $3 \mathrm{~b}$ ). As compared to the binding energy of $\mathrm{Zn} 2 \mathrm{p}$ observed over pure $\mathrm{ZnIn}_{2} \mathrm{~S}_{4}$ (1020.7 and $1043.6 \mathrm{eV}$ ), a higher binding energy shift is observed over $\mathrm{NiS} / \mathrm{ZnIn}_{2} \mathrm{~S}_{4}$ nanocoposite (1021.8 and $1044.7 \mathrm{eV}$ ) (Figure 3c). Similar high binding energy shift is also observed over the high resolution XPS spectra of In 3d (Figure 3d). Such a shift to high binding energy suggests a strong interaction between $\mathrm{ZnIn}_{2} \mathrm{~S}_{4}$ and NiS. It is believed that when $\mathrm{ZnIn}_{2} \mathrm{~S}_{4}$ are connected to NiS, the electron transfer from $\mathrm{ZnIn}_{2} \mathrm{~S}_{4}$ to the more electronegative NiS may result in a decrease of the electron density of $\mathrm{Zn}^{2+}$ and $\mathrm{In}^{3+}$. Therefore the binding energy of $\mathrm{Zn} 2 \mathrm{p}$ and In $3 \mathrm{~d}$ shift to a high binding energy in $\mathrm{NiS} / \mathrm{ZnIn}_{2} \mathrm{~S}_{4}$ nanocoposite. No peaks in the $\mathrm{Ni} 2 \mathrm{p}$ region were observed in the XPS spectra of $0.5 \% \mathrm{NiS} /$ $\mathrm{ZnIn}_{2} \mathrm{~S}_{4}$, probably due to the low amount of NiS loaded and its embed within the $\mathrm{ZnIn}_{2} \mathrm{~S}_{4}$ nanostructures.

The UV-visible diffuse reflectance spectra of NiS/ZnIn ${ }_{2} \mathrm{~S}_{4}$ nanocomposites are displayed in Figure 4. Pure $\mathrm{ZnIn}_{2} \mathrm{~S}_{4}$ has an absorption edge at about $540 \mathrm{~nm}$ with an energy gap estimated to be $2.4 \mathrm{eV}$. Although the loading of NiS onto $\mathrm{ZnIn}_{2} \mathrm{~S}_{4}$ does not obviously change the band gap of $\mathrm{ZnIn}_{2} \mathrm{~S}_{4}$, the resultant $\mathrm{NiS} / \mathrm{ZnIn}_{2} \mathrm{~S}_{4}$ nanocomposites show an enhanced absorption in the visible light region from 550 to $800 \mathrm{~nm}$, attributable to the absorption of NiS.

Photocatalytic hydrogen evolution experiments were carried out over the as-prepared $\mathrm{NiS} / \mathrm{ZnIn}_{2} \mathrm{~S}_{4}$ nanocomposites in the presence of $\mathrm{Na}_{2} \mathrm{~S} / \mathrm{Na}_{2} \mathrm{SO}_{3}$ as sacrificial agent under visible light irradiation. Figure 5 shows the amount of hydrogen evolved over $0.2 \mathrm{wt} \% \mathrm{NiS} / \mathrm{ZnIn}_{2} \mathrm{~S}_{4}$ nanocomposite and is compared with that of pure $\mathrm{ZnIn}_{2} \mathrm{~S}_{4}$ and NiS. Although NiS is a good electrocatalyst for hydrogen evolution [36], no hydrogen was evolved when NiS alone was used as the photocatalyst. Pure $\mathrm{ZnIn}_{2} \mathrm{~S}_{4}$ only had a very low activity with the hydrogen evolution at a rate of $14.1 \mu \mathrm{mol} / \mathrm{h}$. However, the doping of only $0.2 \mathrm{wt} \% \mathrm{NiS}$ onto $\mathrm{ZnIn}_{2} \mathrm{~S}_{4}$ led to its highly enhanced photocatalytic activity for hydrogen evolution. The hydrogen evolution rate over 0.2 wt $\% \mathrm{NiS} / \mathrm{ZnIn}_{2} \mathrm{~S}_{4}$ was enhanced to $70.5 \mu \mathrm{mol} / \mathrm{h}$, about 5 times of that over pure $\mathrm{ZnIn}_{2} \mathrm{~S}_{4}$ under similar condition. This indicates that NiS deposited on the surface on $\mathrm{ZnIn}_{2} \mathrm{~S}_{4}$ can significantly promote the photocatalytic hydrogen evolution over $\mathrm{ZnIn}_{2} \mathrm{~S}_{4}$.

The effect of the amount of NiS loaded on the photocatalytic performance for hydrogen evolution over $\mathrm{ZnIn}_{2} \mathrm{~S}_{4}$ has also been

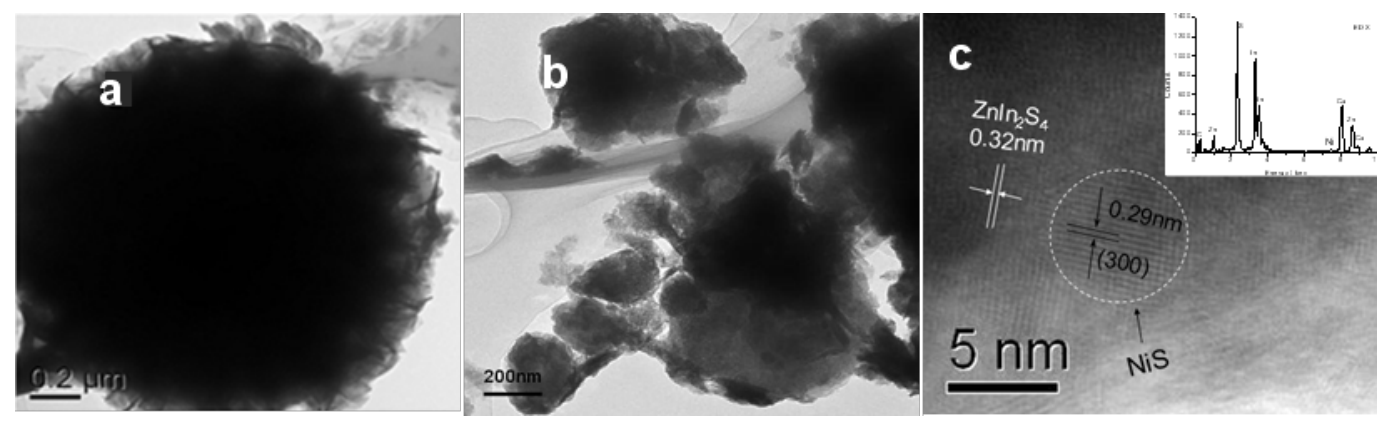

Figure 2: TEM images of (a) $\mathrm{ZnIn}_{2} \mathrm{~S}_{4}$; (b) NiS/Znln $\mathrm{n}_{2}$ and (c) HRTEM image of NiS/Znln $\mathrm{S}_{4}$ (inset: EDS). 

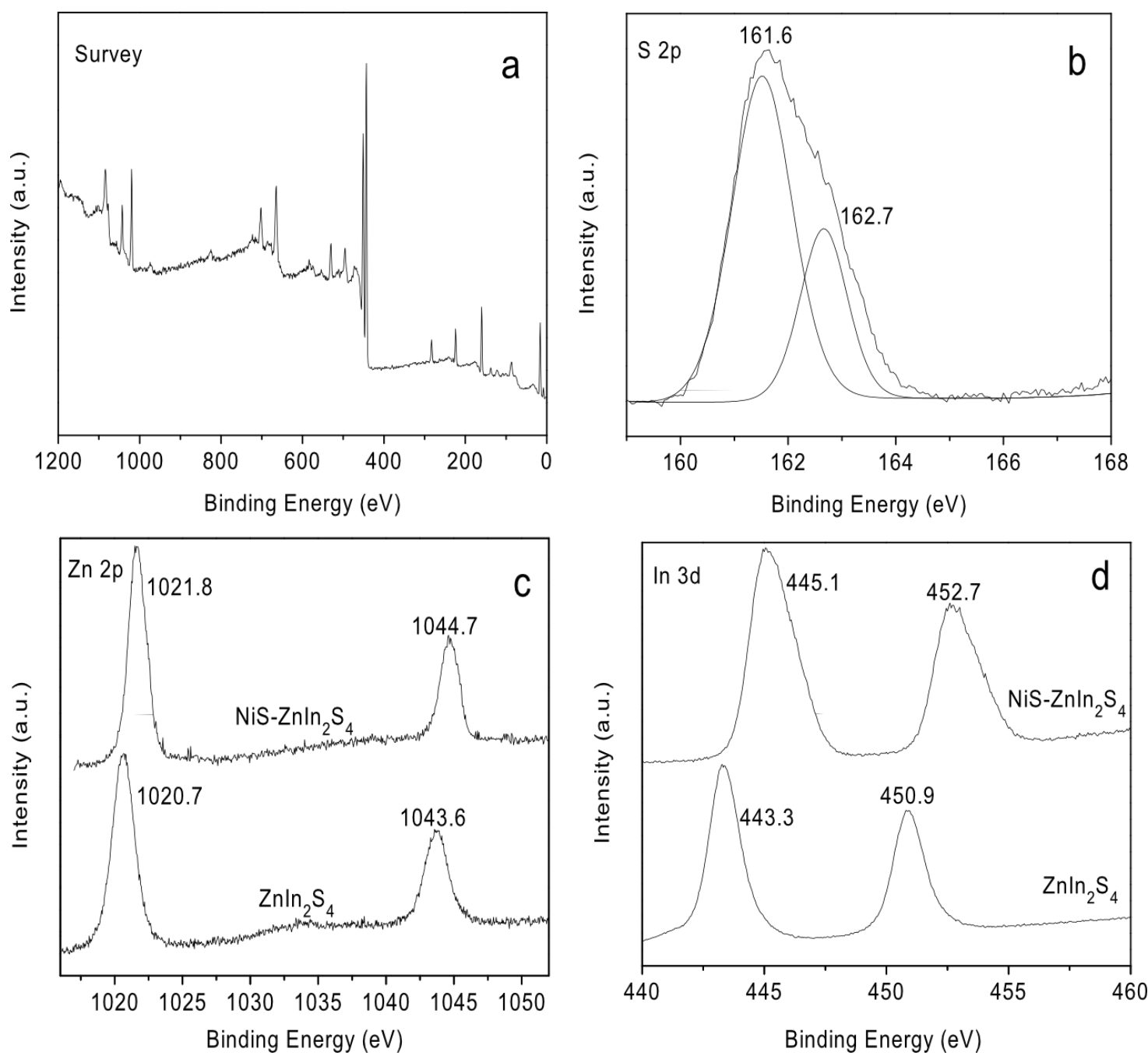

Figure 3: XPS spectra of NiS/Znln ${ }_{2} S_{4}$ and $Z n \ln _{2} S_{4}$ (a) survey spectrum and high-resolution spectra for (b) $S$ 2p; (c) Zn $2 p$ and (d) In $3 d$.

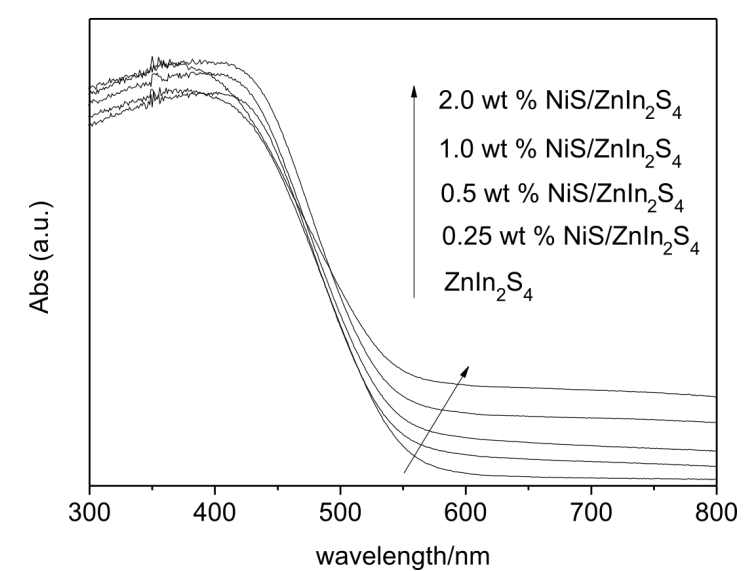

Figure 4: UV-vis diffraction spectra of the pure $\mathrm{Znln}_{2} \mathrm{~S}_{4}$ and 0.25 wt $\%, 0.5$ wt $\%, 1.0$ wt $\%, 2.0$ wt $\% \mathrm{NiS} / Z n \ln _{2} \mathrm{~S}_{4}$.

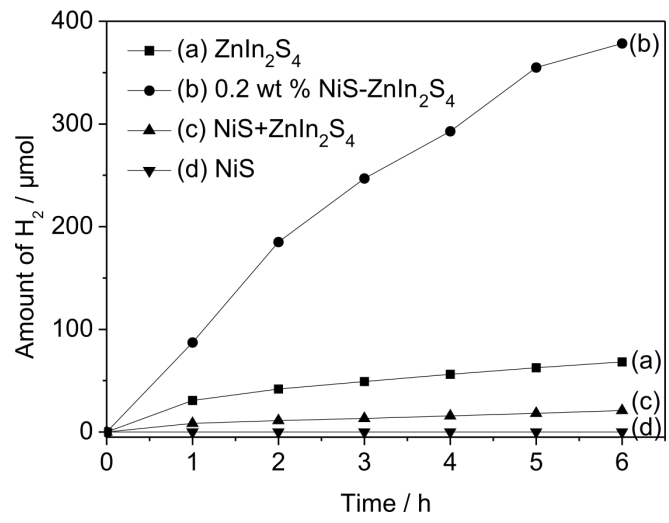

Figure 5: Amount of hydrogen evolution over (a) pure $\mathrm{ZnIn}_{2} \mathrm{~S}_{4}$; (b) 0.2 wt \% $\mathrm{MoS}_{2} / Z \mathrm{ZnIn}_{2} \mathrm{~S}_{4}$; (c) mechanical mixture of NiS $+\mathrm{ZnIn}_{2} \mathrm{~S}_{4}$ and (d) NiS (Reaction conditions: catalyst, $0.05 \mathrm{~g} ; 100 \mathrm{~mL} \mathrm{H} \mathrm{H}_{2} \mathrm{O}$ containing $0.43 \mathrm{M} \mathrm{Na}_{2} \mathrm{~S}$ and $0.5 \mathrm{M} \mathrm{Na}_{2} \mathrm{SO}_{3}$ ). 
investigated and the results are shown in Figure 6. With increasing the amount of loaded NiS on $\mathrm{ZnIn}_{2} \mathrm{~S}_{4}$, the rate of hydrogen evolution on $0.25 \mathrm{wt} \% \mathrm{NiS} / \mathrm{ZnIn}_{2} \mathrm{~S}_{4}$ was enhanced to $98.2 \mu \mathrm{mol} / \mathrm{h}$, about 7 times as that over pure $\mathrm{ZnIn}_{2} \mathrm{~S}_{4}$. An optimum NiS loading amount is found at $0.5 \mathrm{wt} \%$, which exhibits the highest photocatalytic hydrogen evolution rate of $104.7 \mu \mathrm{mol} / \mathrm{h}$, almost 7.4 times as high as that obtained over the NiS-free $\mathrm{ZnIn}_{2} \mathrm{~S}_{4}$. This value is much higher than that observed over $0.5 \mathrm{wt} \% \mathrm{Pt} / \mathrm{ZnIn}_{2} \mathrm{~S}_{4}$ nanocomposite $(77.8 \mu \mathrm{mol} / \mathrm{h})$. A further increase in the amount of NiS resulted in a decrease in the photocatalytic hydrogen evolution rate. Such a decrease in the activity of samples with a heavy loading of NiS is likely due to the shading effect of NiS, which can block the absorption of the incident light by $\mathrm{ZnIn}_{2} \mathrm{~S}_{4}$. Therefore, an appropriate loading amount of NiS is crucial to achieve the optimized photocatalytic activity of the $\mathrm{ZnIn}_{2} \mathrm{~S}_{4}$ photocatalyst.

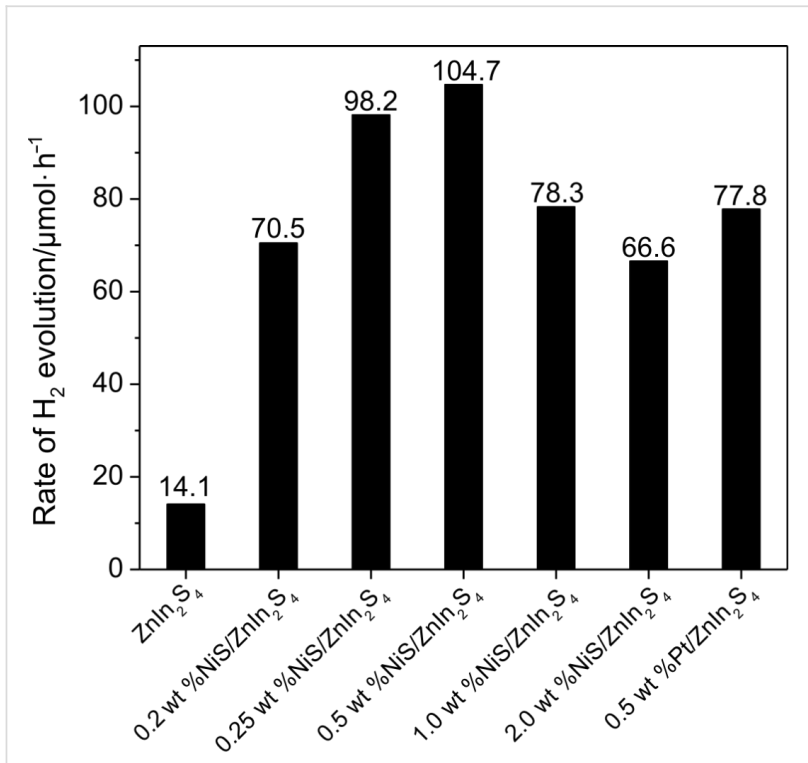

Figure 6: Photocatalytic hydrogen evolution rate over pure $\mathrm{ZnIn}_{2} \mathrm{~S}_{4}$; $\mathrm{ZnIn}_{2} \mathrm{~S}_{4}$ with different amounts of NiS: $0.2 \mathrm{wt} \% ; 0.25 \mathrm{wt} \% ; 0.5 \mathrm{wt} \%$; 1.0 wt $\% ; 2.0$ wt $\%$ and 0.5 wt $\% \mathrm{Pt} / Z \mathrm{ZnI}_{2} \mathrm{~S}_{4}$. (Reaction conditions: catalyst, $0.05 \mathrm{~g} ; 100 \mathrm{~mL} \mathrm{H} \mathrm{H}_{2} \mathrm{O}$ containing $0.43 \mathrm{M} \mathrm{Na}_{2} \mathrm{~S}$ and $0.5 \mathrm{M}$ $\mathrm{Na}_{2} \mathrm{SO}_{3}$ ).

$\mathrm{NiS} / \mathrm{ZnIn}_{2} \mathrm{~S}_{4}$ nanocomposites show high stability during the photocatalytic hydrogen evolution reaction. A prolonged photocatalytic reaction for $15 \mathrm{~h}$ over $0.5 \mathrm{wt} \% \mathrm{NiS} / \mathrm{ZnIn}_{2} \mathrm{~S}_{4}$ revealed that no obvious loss of the activity during the whole reaction period (Figure 7). Besides this, the unchanged XRD pattern of the photocatalyst after the long time reaction also confirms its high stability (Figure 8).

Scheme 1 shows the mechanism proposed for the enhanced photocatalytic hydrogen evolution over NiS/ZnIn $2 \mathrm{~S}_{4}$ nanocomposite. Although the conduction band edge of $\mathrm{ZnIn}_{2} \mathrm{~S}_{4}$

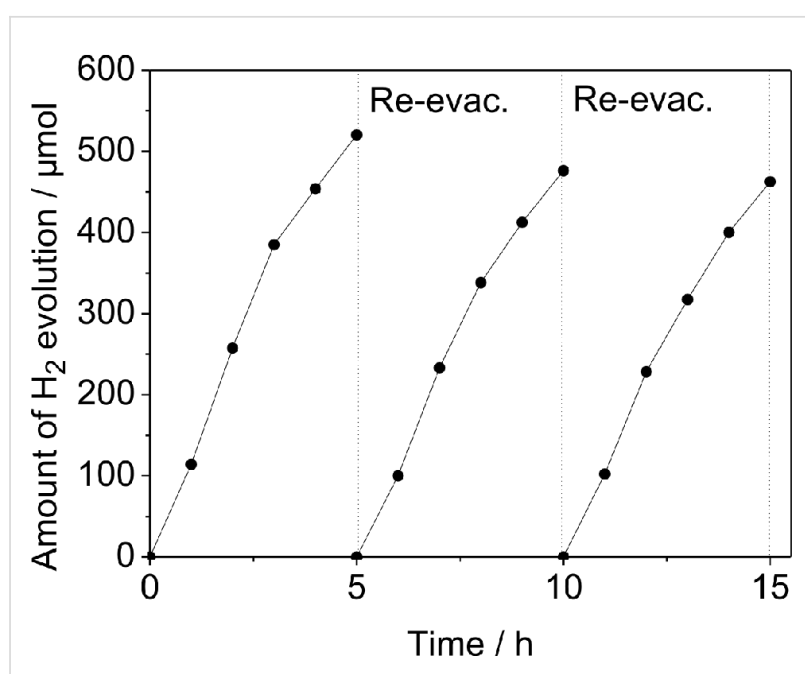

Figure 7: Amount of hydrogen evolved over $0.5 \mathrm{wt} \% \mathrm{NiS} / \mathrm{ZnIn}_{2} \mathrm{~S}_{4}$ system in a $15 \mathrm{~h}$ photocatalytic reaction. (Reaction conditions: catalyst, $0.05 \mathrm{~g} ; 100 \mathrm{~mL} \mathrm{H} 2 \mathrm{O}$ containing $0.43 \mathrm{M} \mathrm{Na}_{2} \mathrm{~S}$ and $0.5 \mathrm{M} \mathrm{Na}_{2} \mathrm{SO}_{3}$ ).

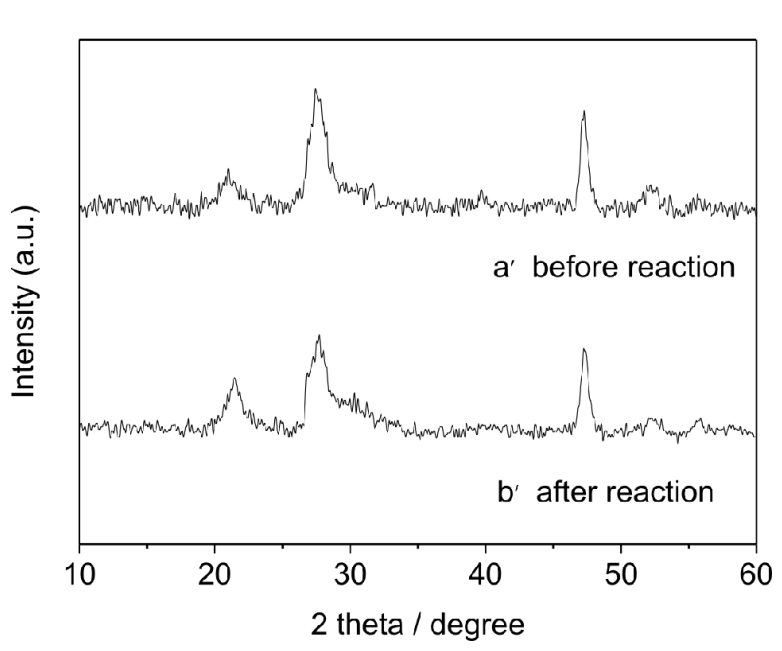

Figure 8: XRD patterns of $0.5 \mathrm{wt} \% \mathrm{NiS} / \mathrm{Znln}_{2} \mathrm{~S}_{4}$ (a) before and (b) after photocatalytic hydrogen evolution reaction.

$(-1.1 \mathrm{eV})$ is higher than the reduction potential of $\mathrm{H}^{+} / \mathrm{H}_{2}$, the rate of hydrogen evolution is low over bare $\mathrm{ZnIn}_{2} \mathrm{~S}_{4}$ due to the rapid recombination rate of photogenerated charge carriers as well as the presence of a large hydrogen evolution overpotential. When NiS is deposited on the surface of $\mathrm{ZnIn}_{2} \mathrm{~S}_{4}$, due to the less negative conduction band of NiS as compared to that of hexagonal $\mathrm{ZnIn}_{2} \mathrm{~S}_{4}$ [43], a directional transfer of the photogenerated electrons from the conduction band of $\mathrm{ZnIn}_{2} \mathrm{~S}_{4}$ to NiS is feasible. Since NiS is a good electrocatalyst for hydrogen evolution [36], NiS can adsorb $\mathrm{H}^{+}$from water and act as the active sites for hydrogen evolution. An efficient electron transfer from the conduction band of $\mathrm{ZnIn}_{2} \mathrm{~S}_{4}$ to NiS in which the hydrogen evolution occurs is crucial for the enhanced hydrogen evolution 
over the NiS/ZnIn ${ }_{2} \mathrm{~S}_{4}$ nanocomposites since controlled experiments performed over a mixture of $\mathrm{NiS}$ and $\mathrm{ZnIn}_{2} \mathrm{~S}_{4}$ shows a much lower photocatalytic activity under similar condition (Figure 5d). Therefore, the formation of a good junction between $\mathrm{ZnIn}_{2} \mathrm{~S}_{4}$ and NiS via the two step hydrothermal processes is important for achieving the highly efficient NiS/ $\mathrm{ZnIn}_{2} \mathrm{~S}_{4}$ nanocomposites with enhanced photocatalytic hydrogen evolution activity.

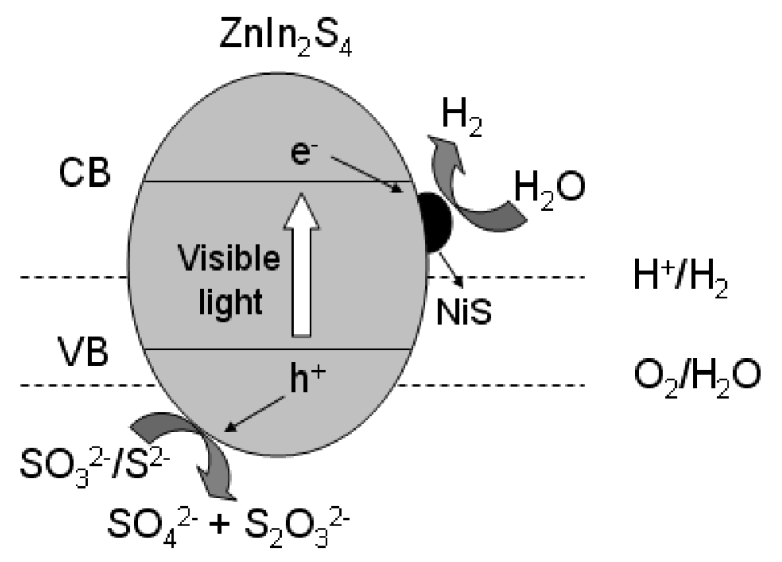

Scheme 1: Schematic illustration of proposed mechanism for photocatalytic hydrogen evolution over $\mathrm{NiS} / \mathrm{ZnIn}_{2} \mathrm{~S}_{4}$ nanocomposite under visible light irradiations.

\section{Conclusion}

In summary, $\mathrm{NiS} / \mathrm{ZnIn}_{2} \mathrm{~S}_{4}$ nanocomposites were facilely synthesized via a two-step hydrothermal method. The as-prepared $\mathrm{NiS} / \mathrm{ZnIn}_{2} \mathrm{~S}_{4}$ nanocomposites showed highly enhanced photocatalytic performance for hydrogen evolution under visible light irradiation. The highest photocatalytic hydrogen evolution rate $(104.7 \mu \mathrm{mol} / \mathrm{h})$, which is even higher than that over $\mathrm{Pt} / \mathrm{ZnIn}_{2} \mathrm{~S}_{4}$ nanocomposite $(77.8 \mu \mathrm{mol} / \mathrm{h})$, was observed over an optimum NiS loading amount of $0.5 \mathrm{wt} \%$. This work demonstrates a high potential of developing the environmental friendly, cheap non-noble-metal co-catalyst for semiconductor-based photocatalytic hydrogen evolution.

\section{Experimental}

Preparations. All the reagents are analytical grade and used without further purifications. Hexagonal $\mathrm{ZnIn}_{2} \mathrm{~S}_{4}$ powder was synthesized according to our previously reported method [16]. In a typical synthesis of $0.5 \mathrm{wt} \% \mathrm{NiS} / \mathrm{ZnIn}_{2} \mathrm{~S}_{4}$ photocatalyst, $0.2 \mathrm{~g} \mathrm{ZnIn}_{2} \mathrm{~S}_{4}, 2.8 \mathrm{mg}$ nickel acetate and $0.9 \mathrm{mg}$ thioacetamide (TAA) were dispersed in $70 \mathrm{~mL}$ of de-ionized water by stirring and ultra-sonication for $2 \mathrm{~h}$. The resultant solution was transferred to a $100 \mathrm{~mL}$ Teflon liner, sealed in the stainless steel autoclave and heated at $120^{\circ} \mathrm{C}$ for $4 \mathrm{~h}$. After the autoclave was cooled to room temperature, the product was collected and washed with de-ionized water several times before it was dried at $60{ }^{\circ} \mathrm{C}$ to obtain the final product. Samples with different amount of $\mathrm{NiS}(0.2,0.25,1.0,2.0 \mathrm{wt} \%)$ were prepared by using different amounts of nickel acetate and TAA precursor during the hydrothermal treatment at $120^{\circ} \mathrm{C}$, while keeping other conditions the same.

0.5 wt $\% \mathrm{Pt} / \mathrm{ZnIn}_{2} \mathrm{~S}_{4}$ was prepared by a photo-deposited method using $\mathrm{H}_{2} \mathrm{PtCl}_{6} \cdot 6 \mathrm{H}_{2} \mathrm{O}$ as the starting material. Pure NiS was prepared by hydrothermal using nickel acetate and TAA as precursors at $120^{\circ} \mathrm{C}$ for $4 \mathrm{~h}$.

Characterizations. X-ray diffraction (XRD) patterns were collected on a Bruker D8 Advance X-ray diffractometer with $\mathrm{Cu} \mathrm{K} \alpha$ radiation. The transmission electron microscopy (TEM) and high-resolution transmission electron microscopy (HRTEM) images were measured by a JEOL model JEM 2010 EX instrument at an accelerating voltage of $200 \mathrm{kV}$. The powder particles were supported on a carbon film coated on a $3 \mathrm{~mm}$ diameter fine-mesh copper grid. A suspension in ethanol was sonicated and a drop was dripped on the support film. X-ray photoelectron spectroscopy (XPS) measurements were performed on a PHI Quantum 2000 XPS system with a monochromatic $\mathrm{Al} \mathrm{K \alpha}$ source and a charge neutralizer. All of the binding energies were referred to the $\mathrm{C} 1 \mathrm{~s}$ peak at $284.8 \mathrm{eV}$ of the surface adventitious carbon. UV-visible diffraction spectra (UV-vis DRS) of the powders were obtained for the dry pressed disk samples using a UV-visible spectrophotometer (Cary 500 Scan Spectrophotometers, Varian). $\mathrm{BaSO}_{4}$ was used as a reflectance standard.

Photocatalytic hydrogen evolution. Photocatalytic hydrogen evolution experiments were carried out in a closed gas circulation and evacuation system fitted with a top Pyrex window. $50 \mathrm{mg}$ of photocatalyst was dispersed in $100 \mathrm{~mL}$ of aqueous solution containing $0.5 \mathrm{M} \mathrm{Na}_{2} \mathrm{SO}_{3}$ and $0.43 \mathrm{M} \mathrm{Na}_{2} \mathrm{~S}$ as sacrificial reagents. The suspension was irradiated with a $300 \mathrm{~W}$ Xe lamp equipped with a $420 \mathrm{~nm}$ cutoff filter to provide the visible light irradiation. The temperature of the reactant solution was maintained at room temperature by a flow of cooling water during the photocatalytic reaction. The amount of hydrogen evolved was determined with an on-line gas chromatography equipped with a TCD detector.

\section{Acknowledgments}

The work was supported by NSFC (21273035), 973 Programs (2014CB239303) and Specialized Research Fund for the Doctoral Program of Higher Education (20123514110002). Z. Li thanks the Award Program for Minjiang Scholar Professorship for financial support. 


\section{References}

1. Chen, X.; Shen, S.; Guo, L.; Mao, S. S. Chem. Rev. 2010, 110 6503-6570. doi:10.1021/cr1001645

2. Tong, H.; Ouyang, S.; Bi, Y.; Umezawa, N.; Oshikiri, M.; Ye, J. Adv. Mater. 2012, 24, 229-251. doi:10.1002/adma.201102752

3. Lewis, N. S.; Nocera, D. G. Proc. Natl. Acad. Sci. U. S. A. 2006, 103, 15729-15735. doi:10.1073/pnas.0603395103

4. Fujishima, A.; Honda, K. Nature 1972, 238, 37-38. doi:10.1038/238037a0

5. Kamegawa, T.; Matsuura, S.; Seto, H.; Yamashita, H. Angew. Chem., Int. Ed. 2013, 52, 916-919. doi:10.1002/anie.201206839

6. Zuo, F.; Bozhilov, K.; Dillon, R. J.; Wang, L.; Smith, P.; Zhao, X.; Bardeen, C.; Feng, P. Angew. Chem., Int. Ed. 2012, 51, 6223-6226. doi:10.1002/anie.201202191

7. Ouyang, S.; Tong, H.; Umezawa, N.; Cao, J.; Li, P.; Bi, Y.; Zhang, Y.; Ye, J. J. Am. Chem. Soc. 2012, 134, 1974-1977. doi:10.1021/ja210610h

8. Seo, S. W.; Park, S.; Jeong, H.-Y.; Kim, S. H.; Sim, U.; Lee, C. W.; Nam, K. T.; Hong, K. S. Chem. Commun. 2012, 48, 10452-10454. doi:10.1039/c2cc36216c

9. Jin, J.; Yu, J.; Liu, G.; Wong, P. K. J. Mater. Chem. A 2013, 1, 10927-10934. doi:10.1039/c3ta12301d

10. Kim, H. N.; Kim, T. W.; Choi, K.-H.; Kim, I. Y.; Kim, Y.-R.; Hwang, S.-J. Chem.-Eur. J. 2011, 17, 9626-9633. doi:10.1002/chem.201100583

11. Tsuji, I.; Shimodaira, Y.; Kato, H.; Kobayashi, H.; Kudo, A. Chem. Mater. 2010, 22, 1402-1409. doi:10.1021/cm9022024

12. Higashi, M.; Domen, K.; Abe, R. J. Am. Chem. Soc. 2013, 135 , 10238-10241. doi:10.1021/ja404030x

13. Wang, Z.; Hou, J.; Yang, C.; Jiao, S.; Huang, K.; Zhu, H. Energy Environ. Sci. 2013, 6, 2134-2144. doi:10.1039/c3ee24370b

14. Ida, S.; Okamoto, Y.; Matsuka, M.; Hagiwara, H.; Ishihara, T. J. Am. Chem. Soc. 2012, 134, 15773-15782. doi:10.1021/ja3043678

15. Wang, X.; Maeda, K.; Thomas, A.; Takanabe, K.; Xin, G.; Carlssom, J. M.; Domen, K.; Antonietti, M. Nat. Mater. 2009, 8, 76-82. doi:10.1038/nmat2317

16. Chen, Y.; Hu, S.; Liu, W.; Chen, X.; Wu, L.; Wang, X.; Liu, P.; Li, Z. Dalton Trans. 2011, 40, 2607-2613. doi:10.1039/c0dt01435d

17. Lei, Z.; You, W.; Liu, M.; Zhou, G.; Takata, T.; Hara, M.; Domen, K.; Li, C. Chem. Commun. 2003, 2142-2143. doi:10.1039/b306813g

18. Shen, S.; Zhao, L.; Guo, L. Int. J. Hydrogen Energy 2008, 33, 4501-4510. doi:10.1016/j.ijhydene.2008.05.043

19. Shen, J.; Zai, J.; Yuan, Y.; Qian, X. Int. J. Hydrogen Energy 2012, 37, 16986-16993. doi:10.1016/j.jhydene.2012.08.038

20. Chai, B.; Peng, T.; Zeng, P.; Zhang, X.; Liu, X. J. Phys. Chem. C 2011, 115, 6149-6155. doi:10.1021/jp1112729

21. Shen, S.; Zhao, L.; Zhou, Z.; Guo, L. J. Phys. Chem. C 2008, 112, 16148-16155. doi:10.1021/jp804525q

22. Mei, Z.; Ouyang, S.; Tang, D.-M.; Kako, T.; Golberg, D.; Ye, J Dalton Trans. 2013, 42, 2687-2690. doi:10.1039/c2dt32271d

23. Chen, Y.; Ge, H.; Wei, L.; Li, Z.; Yuan, R.; Liu, P.; Fu, X. Catal. Sci. Technol. 2013, 3, 1712-1717. doi:10.1039/c2cy20710a

24. Wang, Y.; Wang, Y.; Xu, R. J. Phys. Chem. C 2013, 117, 783-790. doi:10.1021/jp309603c

25. Yang, J.; Yan, H.; Wang, X.; Wen, F.; Wang, Z.; Fan, D.; Shi, J.; Li, C. J. Catal. 2012, 290, 151-157. doi:10.1016/j.jcat.2012.03.008

26. Maeda, K.; Lu, D. L.; Domen, K. ACS Catal. 2013, 3, 1026-1033. doi:10.1021/cs400156m

27. Shimura, K.; Kawai, H.; Yoshida, T.; Yoshida, H. Chem. Commun. 2011, 47, 8958-8960. doi:10.1039/c1cc12287h
28. Tanaka, A.; Sakaguchi, S.; Hashimoto, K.; Kominami, H. ACS Catal. 2013, 3, 79-85. doi:10.1021/cs3006499

29. Iwase, A.; Kato, H.; Kudo, A. Appl. Catal., B: Environ. 2013, 136-137, 89-93. doi:10.1016/j.apcatb.2013.02.006

30. Inoue, Y. Energy Environ. Sci. 2009, 2, 364-386. doi:10.1039/B816677N

31. Ohno, T.; Bai, L.; Hisatomi, T.; Maeda, K.; Domen, K. J. Am. Chem. Soc. 2012, 134, 8254-8259. doi:10.1021/ja302479f

32. Lee, Y.; Teramura, K.; Hara, M.; Domen, K. Chem. Mater. 2007, 19, 2120-2127. doi:10.1021/cm062980d

33. Maeda, K.; Teramura, K.; Masuda, H.; Takata, T.; Saito, N.; Inoue, Y.; Domen, K. J. Phys. Chem. B 2006, 110, 13107-13112. doi:10.1021/jp0616563

34. Hinnemann, B.; Moses, P. G.; Bonde, J.; Jørgensen, K. P.; Nielsen, J. H.; Horch, S.; Chorkendorff, I.; Nørskov, J. K. J. Am. Chem. Soc. 2005, 127, 5308-5309. doi:10.1021/ja0504690

35. Wei, L.; Chen, Y.; Lin, Y.; Wu, H.; Yuan, R.; Li, Z. Appl. Catal., B: Environ. 2014, 144, 521-527. doi:10.1016/j.apcatb.2013.07.064

36. Borucinsky, T.; Rausch, S.; Wendt, H. J. Appl. Electrochem. 1997, 27, 762-773. doi:10.1023/A:1018460425956

37. Zou, Z.; Ye, J.; Sayama, K.; Arakawa, H. Nature 2001, 414, 625-627. doi:10.1038/414625a

38. Kato, H.; Asakura, K.; Kudo, A. J. Am. Chem. Soc. 2003, 125 , 3082-3089. doi:10.1021/ja027751g

39. Zhang, W.; Wang, Y.; Wang, Z.; Zhong, Z.; Xu, R. Chem. Commun. 2010, 46, 7631-7633. doi:10.1039/c0cc01562h

40. Zhang, L.; Tian, B.; Chen, F.; Zhang, J. Int. J. Hydrogen Energy 2012, 37, 17060-17067. doi:10.1016/j.ijhydene.2012.08.120

41. Zhang, J.; Qiao, S. Z.; Qi, L.; Yu, J. Phys. Chem. Chem. Phys. 2013, 15, 12088-12094. doi:10.1039/c3cp50734c

42. Li, N.; Zhou, B.; Guo, P.; Zhou, J.; Jing, D. Int. J. Hydrogen Energy 2013, 38, 11268-11277. doi:10.1016/j.ijhydene.2013.06.067

43. Xu, Y.; Schoonen, M. A. A. Am. Mineral. 2000, 85, 543-556.

\section{License and Terms}

This is an Open Access article under the terms of the Creative Commons Attribution License (http://creativecommons.org/licenses/by/2.0), which permits unrestricted use, distribution, and reproduction in any medium, provided the original work is properly cited.

The license is subject to the Beilstein Journal of Nanotechnology terms and conditions: (http://www.beilstein-journals.org/bjnano)

The definitive version of this article is the electronic one which can be found at: $\underline{\text { doi:10.3762/bjnano.4.107 }}$ 